\title{
Divergent preferences for HIV prevention: A discrete choice experiment for multipurpose HIV prevention products in South Africa
}

Matthew Quaife ${ }^{1,2}$, Robyn Eakle ${ }^{1,2}$, Maria A Cabrera Escobar ${ }^{2}$, Peter Vickerman ${ }^{1,3}$, Maggie KilbourneBrook $^{4}$, Mercy Mvundura ${ }^{4}$, Sinead Delany-Moretlwe ${ }^{2}$, Fern Terris-Prestholt ${ }^{1}$

1 Department of Global Health and Development, London School of Hygiene and Tropical Medicine, London, United Kingdom

2 Wits RHI, University of the Witwatersrand, Johannesburg, South Africa

3 School of Social and Community Medicine, University of Bristol, Bristol, United Kingdom

4 PATH, Seattle, Washington, United States of America

Corresponding Author: Matthew Quaife, London School of Hygiene and Tropical Medicine. matthew.quaife@Ishtm.ac.uk, Tel: (+44) 07855608455

Keywords: HIV prevention, discrete choice experiments, pre-exposure prophylaxis, key populations, South Africa

Word Count: 4,544

Abstract: The development of antiretroviral (ARV)-based prevention products has the potential to substantially change the HIV prevention landscape, yet little is known about how appealing these products will be - compared to existing options - outside of clinical trials. We conducted a discrete choice experiment (DCE) to measure preferences for five new products among four important populations in the HIV response: adult men and women in the general population (aged 18-49), adolescent girls (aged 16-17), and self-identifying female sex workers (aged 18-49). We interviewed 661 self-reported HIV negative participants in peri-urban South Africa, who were asked to choose between three unique, hypothetical products over ten choice sets. Data were analysed using multinomial, latent class and mixed multinomial logit models. HIV protection was the most important attribute to respondents; however, results indicate significant demand among all groups for multipurpose prevention products which offer protection from HIV infection, other STIs, and unwanted pregnancy. All groups demonstrated a strong preference for long-lasting injectable products. There was substantial heterogeneity in preferences within and across population groups. These results suggest that stimulating demand for new HIV prevention products may require a more a nuanced approach than simply developing highly effective products. No one product is likely to be equally attractive or acceptable across different groups. This study strengthens the call for effective and attractive multipurpose prevention products to be deployed as part of a comprehensive combination prevention strategy. 
Ethical Statement: The study was reviewed and approved by the University of the Witwatersrand Human Research Ethics Committee (M140614) and the Research Ethics Committee at the London School of Hygiene and Tropical Medicine (8541-2). All participation in the DCE and supporting qualitative studies was voluntary and subject to completion of a written informed consent process. Adolescent participants were asked for written assent, in addition to written consent provided by a parent or guardian per South African law. A comprehensive distress protocol adopted as standard study procedure ensured that participants who answered in the affirmative to questions about violence and/or other potentially distressing situations were referred to named persons at local clinics and NGOs for additional support.

Funding Sources: Fieldwork was supported by the Bill and Melinda Gates Foundation. MQ receives an Economic and Social Research Council 1+3 studentship. Support for the analysis of this project is made possible by the generous support of the American people through the United States Agency for International Development (USAID) under the terms of the HealthTech $\vee$ Cooperative Agreement \#AID-OAA-A-11-00051. The contents are the responsibility of LSHTM and PATH and do not necessarily reflect the views of USAID or the US Government.

Acknowledgements: We thank all participants for their time and effort completing the survey. We acknowledge the fieldworkers of Progressus Research and Development, ably supported by the management team of Motlalepule Tsepe, Cornelius Monkwe, Lindokuhle Xulu and Reathe RainTaljaard. We acknowledge the valuable input of Maria Sibanyoni, Nyaradzo Mutanha and the great teams of peer educators at Wits RHI and the Center for Positive Care, Ekurhuleni. We thank James Prenter for creating images for the choice tasks, and Duane Blaauw and Mylene Lagarde for helpful comments on the DCE design.

Author Contributions: Conceived, designed and tested the DCE: MQ RE MC PV SDM FTP. Led fieldwork: MQ MC. Analysed the data: MQ FTP PV. Wrote and revised the manuscript: MQ RE MC PV MKB MM SDM FTP. 


\section{Introduction}

South Africa has the largest HIV epidemic in the world, but the health and economic burden of HIV infection is not borne equally. ${ }^{1}$ Women are $40 \%$ more likely to be living with HIV than men, whilst adolescent girls (aged 15-19 years) face an $80 \%$ cumulative lifetime risk of HIV acquisition. ${ }^{2,3}$ Female sex workers (FSW) are designated as a key population for HIV treatment and prevention and estimates suggest that the HIV prevalence among this group in Johannesburg is $72 \%{ }^{4}$

Recent technological developments have led to a number of candidate products for antiretroviral (ARV)-based HIV prevention. ${ }^{5}$ Yet before these are introduced, it is important to understand who will use them to reliably estimate their impact and best plan their introduction. Where different population groups have different preferences for products it is critical that this heterogeneity is identified to ensure that relevant products are made available to potential users. Even if only a small proportion of the population only uses products, they may still be cost-effective if used consistently and effectively among those at risk of HIV acquisition. ARV-based pre-exposure prophylaxis (PrEP) has been shown to offer varying degrees of protection to HIV negative persons when delivered via daily or intermittent oral tablet ${ }^{6,7}$ or long-lasting intravaginal ring ${ }^{8,9}$. However, product protection has been variable during clinical trials as microbicide gels and vaginal rings were found to confer substantially less protection to younger users than to older women primarily due to adherence issues. ${ }^{8,10}$ This is particularly concerning as adolescent girls and young women (AGYW) face a far greater risk of HIV acquisition than older women, or men of any age. ${ }^{3,10}$

A promising field in HIV prevention is the development of multipurpose technologies (MPTs), products which simultaneously provide protection from two or more of HIV, other STIs, and unintended pregnancy. ${ }^{11,12}$ Current MPTs in development include: (1) long-acting drug delivery systems such as intravaginal rings designed to protect from HIV infection and pregnancy (currently in a phase-I trial, ClinicalTrials.gov Identifier: NCT02235662); (2) pericoital drug delivery systems such as vaginal gel, tablets and films designed to protect from HIV; and (3) a combination of products such as a contraceptive diaphragm used with microbicide gel designed to protect from HIV, STIs and pregnancy. ${ }^{11,13}$ Although MPT development is likely to be costly and complex, their benefits may also be large. Firstly, products offering more than one indication may be more attractive to potential users than single purpose products. Secondly, MPT use could crowd-in protection from lesser valued attributes. For example, where users value contraceptive properties more than they do HIV prevention, additional HIV protection would be a positive externality from the use of a dual-protective product. Thirdly, the multipurpose nature of MPTs may reduce the stigma of accessing HIV prevention tools, which has been shown to be a substantive barrier to use, and prevent products from being perceived as for certain populations only. ${ }^{14,15}$

Accounting for the perspectives of end-users in product formulation and delivery could lead to more attractive products, greater uptake and adherence, and increased population protection. This study uses a discrete choice experiment (DCE) to elicit the preferences for five HIV prevention products (oral PrEP, a microbicide gel, a SILCS diaphragm used in concert with gel, an intravaginal ring, and an injectable) from four population groups (adult men and women aged 18-49, adolescent girls aged 1617 and self-identifying female sex workers aged 18-49) in Vosloorus, a township in peri-urban Ekurhuleni, around $30 \mathrm{~km}$ from Johannesburg in the Gauteng province of South Africa. Because there are no observed data for many of these products, we gathered primary stated preference data using the DCE, an end-user focused approach to identifying key determinants of demand. ${ }^{16,17}$ DCEs ask 
respondents to choose their preferred product or service from a set of alternatives over a number (usually between 8-10) of choice tasks. By looking at how respondents choose across repeated scenarios allows researchers to quantitatively elicit the key drivers of decision making. ${ }^{18}$ Although men who have sex with men (MSM) are a high-risk group in South Africa, they were not purposively sampled in this study a) because the contraceptive indication of interest would have little relevance among MSM practicing solely same-sex intercourse, b) the stigma associated with same-sex activity was considered to place interviewers or participants at risk, and c) the small number of MSM likely to be in the geographical location of the survey coupled with the intensive resources required to reach adolescent and FSW groups. Where a small number of MSM were identified the household survey, they were not excluded from data collection or analysis based on reported same-sex activity.

Already popular in the health literature ${ }^{19}$, DCEs are being increasingly applied in the HIV and sexual health fields. Previous work conducted in South Africa found HIV prevention efficacy to be a key driver of demand for a microbicide, a diaphragm or female condom. ${ }^{20}$ A DCE was used to indicate that a rectal microbicide was acceptable among MSM in Thailand, with male sex workers more likely to use an efficacious product than other men. ${ }^{21}$ A DCE in Tanzania showed that different population groups had markedly different preferences for HIV testing interventions among respondents who had been tested previously. ${ }^{22}$ Two DCEs in Malawi found heterogeneity in the preferences of young people for HIV, sexual health, and family planning services. ${ }^{23,}{ }^{24}$ Finally, a conjoint analysis in seven countries found PrEP to be broadly acceptable to potential end-users. ${ }^{25}$

To our knowledge, this is the first study to explore how preferences for new HIV prevention products vary across general and key population groups, and provides important information on a) preferences among different groups, b) variation in preferences within groups, and c) whether new products will be used alongside or instead of condoms. Such "condom migration" has been a concern in the design of PrEP programmes and demonstration projects, yet evidence is mixed as to whether condomless sex will increase after PrEP introduction. ${ }^{6,26-28}$

\section{Methods}

\section{Study context}

Primary data collection was carried out in the town of Vosloorus, around $30 \mathrm{~km}$ south-east of Johannesburg, South Africa. Vosloorus contains a broad range of residential contexts representing a range of demographic, socioeconomic and cultural characteristics. Formative research took place between August and September 2015, and the survey ran from October to December 2015. Further details on study context and methods have been published in the study protocol ${ }^{29}$ and no changes were made to the study design or analysis plan.

\section{Development and design of the discrete choice experiment}

The DCE was developed through an analysis of a previous DCE and focus groups discussions carried out in previous research ${ }^{18,21}$, specifically identifying important characteristics of prevention products and exploring optimal ways to present these in a clear and relatable manner to participants. This was supplemented by a scoping literature review to identify new products and additional attributes which could be important to respondents, which was added to and refined through piloting. We opted to show three alternatives of new products in each task using an unlabelled design where each alternatives represent a generic product within which all characteristics can change as prescribed by 
the statistical design. Though an unlabelled design, were each alternative represents a fixed product category, would have led to greater statistical efficiency, feedback from piloting was that a choice task with five labelled alternatives plus an opt-out was too complex. The opt-out alternative displayed characteristics of a male condom or non-protection, depending on what the respondent reported using in their last sex act.

The DCE was pre-piloted among 17 respondents from across the target populations. A separate design was generated for male and female groups as men can only initiate use of two of the five potential products (the injectable and oral PrEP). The full survey and DCE was then piloted among 45 respondents from the target sampling frame. Few changes were made to the DCE after piloting, except to the side-effects attribute where minor and frequent side-effect symptoms of nausea, stomach cramps, and dizziness were included over major, less frequent side-effects such as reduction in bone mineral density or liver toxicity. This is a limitation of this study, but was necessary due to difficulties in participant comprehension of a) varying probabilities of side-effects and b) medical implications of more serious conditions in a brief DCE interview. Priors from analysis of pilot data $(n=17)$ were used in NGENE software ${ }^{30}$ to generate a single D-efficient design with ten tasks, which avoided dominated or duplicated alternatives as recommended ${ }^{31}$. A D-efficient design was chosen with the aim of improving precision in final model estimates whilst accounting for design constraints arising from restrictions on the frequency that products could be used. The final six attributes of the DCE and their levels are shown in Table 1, and Figure 1 shows an example of how choice tasks were presented to respondents who reported using a condom in their last sex-act. For participants who reported not using a condom at last sex, the final column showed a "nothing" alternative, whilst male respondents saw identical side-effects images but with male characters. The final design incorporated ten choice tasks, with one additional task a repeat to check the consistency of responses.

$<$ Table 1 here $>$

<Figure 1 here>

\section{Survey methods}

A household survey sampled adult men and women (aged 18-49), and adolescent girls (aged 16-17) randomly. Clusters of dwellings (census enumeration areas) within Ekurhuleni were selected at random using municipal data and aerial photographs, and interviewers contacted every second property after a random start. All eligible participants were interviewed within selected households. The DCE and background survey was administered in English, Zulu and Khosa languages.using Open Data Kit (https://opendatakit.org) software on tablet computers, and participants were given ZAR 50 (GBP $£ 2.50$ ) vouchers as compensation for their time. A team of twelve experienced interviewers from a specialist local data collection firm, Progressus Research and Development (http://www.progressus.co.za) implemented the survey.

FSWs are a hard to reach population and we used respondent-driven sampling (RDS) to sample 203 active FSW participants. ${ }^{32}$ Peer educators, most of whom were practicing sex workers, were used to locate 'hotspots' in Ekurhuleni. First, 12 were asked to act as 'seeds' to start RDS chains in different areas (i.e. women working in brothels, hotels or on the street). Seeds were invited to complete the survey and received the same ZAR 50 (GBP f2.50) compensation as participants in the general population. Women were then given four coupons containing study information to distribute to 
colleagues. When each referred colleague attended for interview, their recruiter received a small incentive in the form of a ZAR 20 (GBP $f 1$ ) voucher.

\section{Choice Modelling}

We model choices using random utility models, choosing this approach over alternative methods (random regret minimisation for example) due to its proven consistency in explaining choice behaviour in health applications. We assume that that individual $i(i=1, \ldots, N)$ makes choices such that they maximise utility over the four alternatives presented $(j=1,2,3,4)$. Their axiomatic utility function $U_{i j}$ is decomposed into an explainable systematic component $V_{i j}$ and a random component $\varepsilon_{i j}$, and we specify an indirect utility function for the utility of respondent $i$ from choice $j$ in choice set $c$ as the linear combination of attributes and an error term:

$$
V_{i j c}=X_{i j c} \beta+\varepsilon_{i j c}
$$

With $V_{i j c}$ the utility derived from a choice, $X_{i j c}^{\prime} \beta$ the component of utility that is captured by DCE attributes, and $\varepsilon_{i j c}$ a stochastic (random) component of utility. We specify the vector $X_{i j c}$ as the set of product attributes:

$$
\begin{aligned}
X_{i j c} \beta_{j}=\beta_{0}+ & \beta_{1} \text { Product }_{j}+\beta_{2} \text { HIV }_{j}+\beta_{3} \text { Contraception }_{j}+\beta_{4} \text { STI }_{j}+\beta_{5} \text { Frequency }_{j} \\
& +\beta_{6} \text { SideEffects }_{j}
\end{aligned}
$$

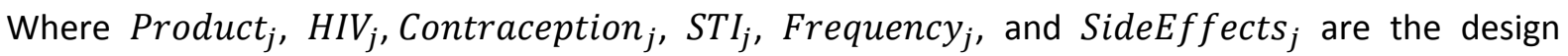
attributes of the DCE, and $\beta_{0}$ a constant. We first estimate equation (1) using a multinomial (or conditional) logit model (MNL) which estimates the probability of individual $i$ choosing alternative $j$ among the set of options $c$ as a probabilistic function of design attributes:

$$
P_{i j c}=\frac{\exp \left(X_{i j c} \beta\right)}{\sum_{J} \exp \left(X_{i j c} \beta\right)}
$$

Although described as the workhorse of discrete choice modelling, the MNL requires two restrictive assumptions: the IIA assumption of independence of irrelevant alternatives (concordant with the IID distribution of the disturbance), and homogenous preferences across individuals where every individual is assumed to have the same tastes as the sample average, which does not allow us to uncover differences in preferences that are important in guiding policy. ${ }^{18}$ We assume that individuals choose the service associated with the highest utility such that the probability that individual $i$ chooses alternative $j$ over $k$ is given as:

$$
\operatorname{Pr}_{j i}=\operatorname{Pr}\left(U_{j i}>U_{k i}\right)=\operatorname{Pr}\left(V_{j i}+\varepsilon_{j i}>V_{k i}+\varepsilon_{k i}\right)=\operatorname{Pr}\left(V_{j i}-V_{k i}>\varepsilon_{j i}-\varepsilon_{k i}\right)
$$

In this paper, as different MNL models are estimated for each population, the assumption required is homogeneity of preferences within each group. As groups are more homogenous in nature due to separate inclusion criteria, assuming identical preferences across members within each group may be less restrictive than assuming this across the range of populations in our sample.

Nevertheless, to recognise that individuals within groups may have different preferences, we apply a random parameter logit (MMNL or mixed logit random parameter) model. The MMNL allows for 
preference (taste) heterogeneity within groups so does not require the IIA assumption to hold. Briefly, the MMNL extends the MNL model by decomposing the error term into two components:

$$
U_{i j}=V_{i j}+\left[\Gamma_{i j}+\varepsilon_{i j}\right]
$$

Which, when the distribution of the random component $\Gamma_{i j}$ is specified by the analyst, is used to estimate individual-level coefficients:

$$
\beta_{i k}=\beta_{k}+\delta_{k} Z_{i}+\Gamma_{k} v_{i k}
$$

Where $\delta_{k} Z$ reflects observable heterogeneity, and $\Gamma_{k} v_{i k}$ unobservable heterogeneity.

Finally, we use a latent class approach to explore unobserved heterogeneity in choice data. A semiparametric approach, a latent class model assumes the existence of underlying subgroups (classes) of respondents whose membership of each class is characterised by unobserved, or latent, variables. A posterior probability is assigned to each participant for membership to each class, and when participants are allocated to classes based on their highest probability it is possible to compare the observable characteristics of participants across classes ${ }^{18,34}$.

Latent class modelling offers a different approach to relaxing the IIA assumption of the MNL model. Furthermore the basic latent class model does not require parametric distributional assumptions to be imposed by the analyst, whilst results have been shown less sensitive to computational variations in estimation, such as starting values or optimisation algorithms which can vary across software packages. ${ }^{35,36} \mathrm{~A}$ latent class approach estimates separate parameter vectors for different classes of the sample with MNL models. The model relaxes the IIA assumption by assuming that preferences are homogenous within, but not across, classes. Thus the probability of respondent $i$ choosing alternative $j$ in choice set $c$ conditional on class membership $k$ is:

$$
P_{i c}\left(J \mid \beta_{k}\right)=\sum_{k=1}^{K} \pi_{i k} \frac{\exp \left(X_{i j c} \beta_{k}\right)}{\sum_{J} \exp \left(X_{i j c} \beta_{k}\right)}
$$

The probability of respondent $i$ belonging to class $k$ is $\pi_{i k}$. Class membership is unobservable, however, we can regress the probability of class membership on a set of observable characteristics such that:

$$
\pi_{i k}=\frac{\exp \left(Z_{i}^{\prime} \delta_{k}\right)}{\sum_{k=1}^{K} \exp \left(Z_{i}^{\prime} \delta_{k}\right)}
$$

With $Z_{i}$ a vector of individual characteristics, and $\delta_{k}$ a vector of parameters to be estimated.

We run latent class logit models on the male sample, alongside a pooled sample of all three female groups. This approach removes the implicit assumption that women's preferences will differ by their sample category, and instead allows the data to drive evidence of variation and similarity among subgroups. By pooling we lose generalisability from the results of this specification; however, results from the pooled analysis are useful to identify important subgroups of the female population. We assess the optimal number of classes through comparison of model fit via log-likelihood function and Akaike Information Criteria (AIC). 


\section{Heterogeneity}

We use two approaches to explore heterogeneity in preferences. First, we examine the distribution parameters of the MMNL model to see where variation remains in addition to that captured by the random parameters. Secondly, we estimate the latent class model (equation 8) and explore how preferences vary by class, specifying several observable characteristics (Error! Reference source not found.) which influence the probability of class membership.

Our rationale for including these observable characteristics is as follows. Age is strongly associated with HIV and pregnancy risk ${ }^{37,38}$, yet little is known about the effect of age on preferences for protection against HIV or pregnancy. Structural drivers are legal, economic and social factors which shape HIV risk. ${ }^{39}$ Exposure to intimate partner violence (IPV) has been shown to be associated with increased HIV risk in many contexts ${ }^{40,41}$, whilst limited livelihood opportunities, and unrestricted alcohol availability alongside drinking norms are also significant factors in HIV risk. ${ }^{42-45}$ Finally, life circumstances play an important role in the extent to which people place themselves at, or are able to protect themselves from, HIV risk. ${ }^{46,47}$ We therefore explore attitudes towards pregnancy among women and how preferences may be affected by male circumcision, which reduces the likelihood of a male acquiring HIV. ${ }^{48,49}$ Finally, we explore associations with protective attributes, having an external partner in the previous three months, and having high HIV knowledge as assessed by the number of correct answers on a knowledge tool. ${ }^{50}$

\section{Model estimation}

Models were estimated in NLOGIT $5^{51}$ through a maximum likelihood approach and MMNL models were estimated using 500 Halton draws with start values obtained from a MNL model. All parameters were generic across three unlabelled alternatives, with labels entering the DCE through an attribute level. All design attributes were specified as random with parameters following a normal distribution to characterise our uncertainty in the nature and direction of heterogeneity around coefficients. Triangular and lognormal distributions were tested and did not change the sign or significance of the main effects. General population samples were analysed with survey weighting such that age and sex characteristics matched those of the general population. FSW RDS data were weighted using age characteristics using RDSAT software. ${ }^{52}$ All attribute levels were effects coded ${ }^{1}$, except for HIV protection which was estimated linearly..$^{53}$

\section{Results}

\section{Sample characteristics and generalisability}

In the household survey, of 2271 persons identified door-to-door, 650 were eligible (29\%). Of those, $30(5 \%)$ were not found after five attempts and $11(2 \%)$ refused to be interviewed, leading to 609 (94\%) completed interviews. In total 661 interviewees met the DCE eligibility criteria across the four populations. Notably, 126 (62\%) of adolescent girls reported not being sexually active whilst selfreported HIV prevalence was broadly comparable across and within populations to that of other representative surveys., ${ }^{34}$ The inclusion criteria for adolescent girls did not require sexual activity because adolescent girls face substantial and immediate risk of HIV at sexual debut, whilst HIV

\footnotetext{
${ }^{1}$ Effects coding is similar to dummy coding in that it allows for non-linear effects in attribute levels, but does not perfectly confound the base attribute level with the grand mean of the utility function. Coefficients are therefore interpreted as divergence from the mean for that attribute
} 
prevention programmes do not target based on reported sexual activity. The preferences of nonsexually active adolescent are important for ensuring that attractive products are made available at the earliest possible exposure to HIV risk. In addition, if sexual activity was an explicit inclusion criteria for adolescents, confidentiality could have been breached if other members of the household knew that an interview did, or did not, take place.

$<$ Table 2 here $>$

Table 2 displays the descriptive statistics for eligible respondents in each population. Comparability is limited across groups due to varying inclusion criteria for adolescent girls and FSWs, the latter also sampled through RDS. The mean age across adult groups was similar and whilst a large number of respondents in each group reported being in a relationship, many fewer reported cohabiting with a sexual partner. Reported lifetime sexual partners among adult males (13.06) were higher than among adult females (4.55). Reported condom use at last sex with a regular partner was significantly lower among adult females (43\%) than any other group, though this figure is comparable with that of $34 \%$ from a nationally representative survey. ${ }^{3,4}$

The DCE was well understood by participants; just $2 \%$ of respondents reported finding the DCE quite difficult or very difficult, whilst $81 \%$ of respondents chose the same alternative twice in the repeated choice task. $61 \%$ of respondents chose the product (or one of the products when values were duplicated) with the highest HIV protection in every choice set.

\section{Analysis of preference data}

$<$ Table 3 here $>$

Table 3 shows MMNL results and reports the coefficients of each attribute level parameter. Results from the MNL model are given in supplementary table 2 . Where coefficients are significantly greater than zero, an attribute can be interpreted as having a relatively positive impact on participant utility. The magnitude of coefficients can be directly compared within each model. The significance of coefficients (i.e. what is important to people's choices) can be compared across populations, but due to possible differences in the scale of choice data across groups, coefficients cannot be directly compared across populations. In both specifications and across all groups HIV protection was the strongest determinant of choice, whilst protection against pregnancy and other STIs was also significantly attractive. The absence of side-effects was important to adult men but not to any female groups, although FSWs found nausea significantly worse than other side-effects. There was no clear pattern of demand around frequency of use across groups.

There are some differences between the product coefficients in the MNL and MMNL models, indicating that tastes vary within populations - this is also highlighted by the positive distribution parameters. In both specifications adult women and FSWs significantly dislike oral PrEP and favour injectable products, with FSWs additionally disliking microbicide gels. Among adolescent girls, the MNL indicates a significant dislike for the vaginal ring which is insignificant in the MMNL model, whilst the MMNL model shows the same pattern of statistically significant preferences for oral PrEP and injectable products as other female groups. 


\section{Preference Heterogeneity}

\section{Latent class specification \\ $<$ Table 4 here $>$}

Latent class models are presented for female and male respondents in panels A and B of Table 4 respectively, and were computed without survey weighting. HIV knowledge (as assessed through the a tool presented in Supplementary Material 1 ) is a key driver of demand for HIV protection as Class 1 members (34\% of the female sample) show strong preferences for STI and pregnancy protection but do not value HIV protection in a new product. Class membership is additionally predicted by being of younger age, a FSW, or an adolescent, indicating that there is significant variation in preferences among female groups. A HIV preventive MPT would offer substantial benefit to this group of women, as contraceptive or STI preventative products could be bundled with HIV protection, offering additional protection.

In contrast, members of class 2 ( $19 \%$ of the sample) display very strong preferences for HIV protection, valuing it more than twice as highly as other classes. Class 2 members also value pregnancy protection, but do not find STI protection appealing. Class 2 appears to be a high-risk group as, relative to class 3 , members are more likely to have used alcohol the last time they had sex, and have lower HIV knowledge. Finally, class 2 members are more likely to be FSW or adolescent girls than class 3 members, and indicate a dislike for coitally-specific products. Class 3 members ( $48 \%$ of the sample) strongly value protection from HIV, pregnancy and STIs, and indicate a dislike for the vaginal ring product. This group is likely to comprise of adult women in the general population.

Among adult males, membership of either class (42\%) is not significantly predicted by the characteristics we define, indicating that drivers of preference heterogeneity differ between men and women and that male preferences are not shaped by structural risks in the same manner as female preferences. However there is still evidence of heterogeneity in preferences among adult men. The most notable variation between the two groups is the non-significance of the pregnancy prevention parameter among class 2 men indicating that, in order to make HIV prevention products attractive to these men, potential HIV and STI prevention properties should be emphasised. Men in class 1 strongly prefer oral PrEP, whilst the strongly significant opt-out parameters in both groups show an inclination to continue current practice suggesting that the expected reporting bias towards switching to new products may not be as strong as hypothesised.

\section{Marginal effects}

DCEs estimate the relative strength of preferences, and therefore we cannot simply compare the magnitude of coefficients across groups. However, the ratios of coefficients are comparable across populations and show the variation in trade-offs across groups. The marginal effects of factors relative to the common denominator of HIV protection are provided in supplementary table 3, computed from the coefficients in table 3. Adult men are only willing to forgo $3 \%$ HIV protection for a contraceptive product, but adult women are willing to forgo $11 \%$. Results suggest that all female groups value the male condom substantially more than men, and that preferences for STI protection is broadly comparable to pregnancy protection across female groups. Men value the absence of side-effects much more than women and FSWs value STI protection almost three times greater than any other group. 


\section{Discussion}

This study assessed preferences for new HIV prevention products among adult men and women, adolescent girls, and female sex workers. Consistent with prior expectations HIV protection was the most important attribute to respondents, particularly those with high levels of HIV knowledge, however, results suggest that demand would increase for all groups if multipurpose protection was incorporated. There was relatively little variation in preferences for oral PrEP, microbicide gel, the SILCS diaphragm and the vaginal ring when compared to strong preferences for a long-lasting injectable ARV. This could be influenced by the strong awareness and use of injectable contraceptives in South Africa. ${ }^{54}$

This study also provides evidence to explain data from clinical trials of new HIV prevention products. Neither adult women nor adolescent girls found the vaginal ring appealing, whilst an injectable product was favoured by all groups. In the context of trial data suggesting that younger women were not able to adhere to vaginal ring use to the same extent as older women ${ }^{8,9}$, these results suggest that younger women in particular do not favour a vaginal ring prevention system. Incorporating multipurpose properties to a vaginal ring could mitigate some of the intrinsic dislike of this product, however, these data suggest that a vaginal ring product will not be an attractive offering for younger populations.

Importantly this study suggests that, in order to fully catch the attention of AGYW, products must not be single-purpose but provided to offer multipurpose prevention against HIV, pregnancy, and other STIs. AGYW have been identified as key populations in HIV prevention, and it is critical that new biomedical products are made to be attractive and easy for these women to use. ${ }^{3}$ Although MPT products are generally at the concept stage of development, bundling PrEP with other sexual and reproductive health services may make it more attractive than vertical programmes until such time as an MPT is licensed.

Although there are some similarities across groups, the divergence in preferences shown here can provide useful information for programming. Adolescent and adult females are unlikely to be put off by minor side-effects and demonstrate comparable, statistically significant preferences for STI and pregnancy protection. Adult women, however, are much more likely to find the male condom appealing relative to new products, indicating that demand for ARV-based prevention may be less among older, lower-risk women who may prefer on-demand methods. Products rolled out into higherrisk groups (such as AGYW) are more likely to be cost-effective - as long as they can be used consistently and effectively. ${ }^{55}$ Findings from the latent class analysis show notable segments in the market for PrEP, with some women very concerned about HIV and STI protection, and others who are not at all. In addition, female sex workers demonstrate a much stronger preference for STI protection than any other group, perhaps because STI acquisition is more likely to immediately affect earnings than HIV or pregnancy. ${ }^{56}$

Finally, these results suggest that a one size-fits all HIV prevention package will not be an effective or efficient use of resources. This is consistent with recent trajectories of contraceptive and HIV research where it has been recognised that a range of options can optimise uptake and adherence. ${ }^{57}$ Because groups have different preferences and needs, further highlighted by these results, there is a strong case for greater tailoring of prevention services, for at risk young women in particular. 
This study has some limitations. Firstly, DCEs are hypothetical tools and may not correlate perfectly with real-world choices. However, recent evidence shows that DCEs conducted in health fields predict real world choices with a relatively high sensitivity, suggesting a reasonable degree of external validity. ${ }^{58}$ Overall, the random sample of the general population survey design and the similarity of sample descriptive statistics with other representative surveys mean findings may have relevance for other South African settings. Self-reported rates of condom use among males and adolescent girls were higher than other representative surveys and may indicate acceptability biases in reporting, or that condoms were more readily available to this group than others. This study did not include a price attribute, and therefore does not enable the calculation of willingness to pay estimates for different products. However, given the South African context where prevention products will likely be free-ofcharge to potential users in the public sector, including a price element would have made the choice task unrealistic. Product profiles in the DCE do not perfectly match with candidate products in development, for example injectable trials are based on administration every eight weeks rather than every four or twelve explored in this DCE, whilst rare but serious side-effects were not included in the choice tasks. In addition, injectable products may be more likely to initiate drug resistance as they have been shown to remain in the body at a low leve ${ }^{59}$, and this is not included here. Finally, there is a lively literature discussing the reliability of RDS to generate generalisable samples suggesting that, at worst, RDS is a superior form of convenience sampling for hard-to-reach populations and, at best, produces bias-free and generalisable samples. ${ }^{32,60}$

Further research is needed to estimate the impact and cost-effectiveness of potential single- and multi-purpose HIV prevention products. If models do not incorporate the variation in product preferences that we observe here and instead assume an average uptake across groups, they may give misleading results. Because models are used to generate impact predictions for investment cases in low- and middle-income countries (e.g. in South Africa ${ }^{61}$ ), inaccurate predictions could result in reduced efficiency in the allocation of resources, and large opportunity costs in terms of benefits forgone. This paper shows that particular attention should be paid to the perspectives of different groups of end-users.

\section{Conclusion}

This study involved a discrete choice experiment among four groups in Ekurhuleni Municipality in South Africa. In general, respondents indicated a strong desire for products that are highly effective in preventing HIV infection. However, there was strong demand across all groups for multipurpose prevention products to protect against other STIs and pregnancy. Further analysis shows substantive heterogeneity across and within groups, suggesting that a variety of prevention methodologies are required to meet the demands of different groups. These results strengthen the call for effective and attractive multipurpose prevention technologies to be deployed as part of a comprehensive combination prevention strategy.

\section{References}

1. World Health Organisation. Guidelines on When to Start Anti-Retroviral Therapy and on PreExposure Prophylaxis for HIV, 2015.

2. Tanser F, Bärnighausen T, Cooke GS, Newell M-L. Localized spatial clustering of HIV infections in a widely disseminated rural South African epidemic. International Journal of Epidemiology 2009: dyp148. 
3. Shisana O, Rehle T, Simbayi L, et al. South African National HIV Prevalence, Incidence and Behaviour Survey 2012. Cape Town: HSRC Press, 2014.

4. UCSF, Anova Health Institute, WRHI. South African Health Monitoring Study (SAHMS), Final Report: The Integrated Biological and Behavioural Survey among Female Sex Workers, South Africa 2013-2014. San Francisco UCSF, 2015.

5. AVAC. HIV Prevention Research and Develoment Database. 2015. http://www.avac.org/pxrd (accessed 2/11/2016.

6. Molina J-M, al. e. On Demand PrEP With Oral TDF-FTC in MSM: Results of the ANRS Ipergay Trial. . Conference on Retroviruses and Opportunistic Infections (CROI); 2015; Seattle, USA; 2015.

7. McCormack S, al. e. Pragmatic Open-Label Randomised Trial of Pre-Exposure Prophylaxis: the PROUD study. Conference on Retroviruses and Opportunistic Infections (CROI); 2015; Seattle, USA; 2015.

8. Nel A, Kapiga S, Bekker L-G, et al. Safety and Efficacy of Dapivirine Vaginal Ring for HIV-1 Prevention in African Women. CROI 2016. Seattle, USA; 2016.

9. Baeten JM, Palanee-Phillips T, Brown ER, et al. A Phase III Trial of the Dapivirine Vaginal Ring for HIV-1 Prevention in Women. Conference on Retroviruses and Opportunistic Infections Boston, MA; 2016.

10. van der Straten A, Van Damme L, Haberer JE, Bangsberg DR. Unraveling the divergent results of pre-exposure prophylaxis trials for HIV prevention. AIDS 2012; 26(7): F13-F9.

11. Friend DR. An update on multipurpose prevention technologies for the prevention of HIV transmission and pregnancy. Expert opinion on drug delivery 2016; 13(4): 533-45.

12. Brady M, Tolley E. Aligning product development and user perspectives: social-behavioural dimensions of multipurpose prevention technologies. BJOG : an international journal of obstetrics and gynaecology 2014; 121 Suppl 5: 70-8.

13. Friend DR, Doncel GF. Combining prevention of HIV-1, other sexually transmitted infections and unintended pregnancies: Development of dual-protection technologies. Antiviral research 2010; 88 Suppl 1: S47-54.

14. Das U, Sharma M, Kilbourne-Brook M, Coffey PS. Exploring vaginal ring acceptability for contraception and sexually transmissible infection protection in India: a qualitative research study. Sex Health 2015; 12(6): 532-40.

15. MacPhail C, Terris-Prestholt F, Kumaranayake L, Ngoako P, Watts C, Rees H. Managing men: women's dilemmas about overt and covert use of barrier methods for HIV prevention. Culture, health \& sexuality 2009; 11(5): 485-97.

16. Ryan M, Gerard K, Amaya-Amaya Me. Using Discrete Choice Experiments to Value Health and Health Care. 2008// 2008.

17. Bridges JFP, Hauber AB, Marshall D, et al. Conjoint Analysis Applications in Health-a Checklist: A Report of the ISPOR Good Research Practices for Conjoint Analysis Task Force. Value in Health 2011; 14(4): 403-13.

18. Hensher D, Rose J, Greene W. Applied Choice Analysis: Second Edition. Cambridge: Cambridge University Press; 2015.

19. de Bekker-Grob EW, Ryan M, Gerard K. Discrete choice experiments in health economics: a review of the literature. Health economics 2012; 21(2): 145-72.

20. Terris-Prestholt F, Hanson K, MacPhail C, Vickerman P, Rees H, Watts C. How much demand for New HIV prevention technologies can we really expect? Results from a discrete choice experiment in South Africa. PLoS One 2013; 8(12): e83193.

21. Newman PA, Cameron MP, Roungprakhon S, Tepjan S, Scarpa R. Acceptability and Preferences for Hypothetical Rectal Microbicides among a Community Sample of Young Men Who Have Sex with Men and Transgender Women in Thailand: A Discrete Choice Experiment. AIDS and behavior 2015.

22. Ostermann J, Njau B, Brown DS, Mühlbacher A, Thielman N. Heterogeneous HIV Testing Preferences in an Urban Setting in Tanzania: Results from a Discrete Choice Experiment. PLOS ONE 2014; 9(3): e92100. 
23. Michaels-Igbokwe C, Lagarde M, Cairns J, the Integra I, Terris-Prestholt F. Designing a package of sexual and reproductive health and HIV outreach services to meet the heterogeneous preferences of young people in Malawi: results from a discrete choice experiment. Health economics review 2015; 5: 9.

24. Michaels-Igbokwe C, Terris-Prestholt F, Lagarde M, Chipeta E, Cairns J. Young People's Preferences for Family Planning Service Providers in Rural Malawi: A Discrete Choice Experiment. PLoS One 2015; 10(12): e0143287.

25. Eisingerich AB, Wheelock A, Gomez GB, Garnett GP, Dybul MR, Piot PK. Attitudes and Acceptance of Oral and Parenteral HIV Preexposure Prophylaxis among Potential User Groups: A Multinational Study. PLOS ONE 2012; 7(1): e28238.

26. Underhill K. Study designs for identifying risk compensation behavior among users of biomedical HIV prevention technologies: balancing methodological rigor and research ethics. Social science \& medicine (1982) 2013; 94: 115-23.

27. Blumenthal J, Haubrich R. Risk Compensation in PrEP: An Old Debate Emerges Yet Again. The virtual mentor: VM 2014; 16(11): 909-15.

28. Foss AM, Vickerman PT, Heise L, Watts $\mathrm{CH}$. Shifts in condom use following microbicide introduction: should we be concerned? Aids 2003; 17(8): 1227-37.

29. Quaife M, Eakle R, Cabrera M, et al. Preferences for ARV-based HIV prevention methods among men and women, adolescent girls and female sex workers in Gauteng Province, South Africa: a protocol for a discrete choice experiment. BMJ open 2016; 6(6): e010682.

30. ChoiceMetrics. Ngene version 1.1.2. Sydney, Australia; 2012.

31. Johnson FR, Lancsar E, Marshall D, et al. Constructing experimental designs for discrete-choice experiments: report of the ISPOR conjoint analysis experimental design good research practices task force. Value in Health 2013; 16(1): 3-13.

32. Johnston LG, Sabin K. Sampling Hard-to-Reach Populations with Respondent Driven Sampling. Methodological Innovations Online 2010; 5(2): 38-48.

33. McFadden D. Conditional logit analysis of qualitative choice behaviour. In: Zarembka P, ed. Frontiers in econometrics. New York: Academic Press; 1974.

34. Mandeville KL, Ulaya G, Lagarde M, Muula AS, Dzowela T, Hanson K. The use of specialty training to retain doctors in Malawi: A discrete choice experiment. Social science \& medicine (1982) 2016; 169: 109-18.

35. Chang JB, Lusk JL. Mixed logit models: accuracy and software choice. Journal of Applied Econometrics 2011; 26(1): 167-72.

36. Chiou L, Walker JL. Masking identification of discrete choice models under simulation methods. Journal of Econometrics 2007; 141(2): 683-703.

37. Celum CL, Delany-Moretlwe $\mathrm{S}$, McConnell M, et al. Rethinking HIV prevention to prepare for oral PrEP implementation for young African women. J Int AIDS Soc 2015; 18(4).

38. Spiegelhalter D. Sex by numbers: what statistics can tell us about sexual behaviour: Profile Books; 2015.

39. STRIVE Consortium. What are structural drivers? 2016. http://strive.Ishtm.ac.uk/ (accessed 29/11/2016.

40. Beattie TS, Bhattacharjee $P$, Ramesh BM, et al. Violence against female sex workers in Karnataka state, south India: impact on health, and reductions in violence following an intervention program. BMC Public Health 2010; 10: 476.

41. Seeley J, Watts CH, Kippax S, Russell S, Heise L, Whiteside A. Addressing the structural drivers of HIV: a luxury or necessity for programmes? Journal of the International AIDS Society 2012; 15(Suppl 1).

42. Mbonye $\mathrm{M}$, Rutakumwa $\mathrm{R}$, Weiss $\mathrm{H}$, Seeley J. Alcohol consumption and high risk sexual behaviour among female sex workers in Uganda. African journal of AIDS research : AJAR 2014; 13(2): 145-51. 
43. Nglazi MD, van Schaik N, Kranzer K, Lawn SD, Wood R, Bekker L-G. An incentivized HIV counseling and testing program targeting hard-to-reach unemployed men in Cape Town, South Africa. Journal of acquired immune deficiency syndromes (1999) 2012; 59(3): e28.

44. Eaton LA, Kalichman SC. Risk compensation in HIV prevention: Implications for vaccines, microbicides, and other biomedical HIV prevention technologies. Curr HIV/AIDS Rep 2007; 4(4): 16572.

45. Kalichman SC, Simbayi LC, Kaufman M, Cain D, Jooste S. Alcohol use and sexual risks for HIV/AIDS in sub-Saharan Africa: systematic review of empirical findings. Prevention science 2007; 8(2): 141-51.

46. Finocchario-Kessler S, Mabachi N, Dariotis JK, Anderson J, Goggin K, Sweat M. "We weren't using condoms because we were trying to conceive": The need for reproductive counseling for HIVpositive women in clinical care. AIDS patient care and STDs 2012; 26(11): 700-7.

47. Crankshaw TL, Matthews LT, Giddy J, et al. A conceptual framework for understanding HIV risk behavior in the context of supporting fertility goals among HIV-serodiscordant couples. Reproductive health matters 2012; 20(39): 50-60.

48. Weiss HA, Quigley MA, Hayes RJ. Male circumcision and risk of HIV infection in sub-Saharan Africa: a systematic review and meta-analysis. Aids 2000; 14(15): 2361-70.

49. Mills E, Cooper C, Anema A, Guyatt G. Male circumcision for the prevention of heterosexually acquired HIV infection: a meta-analysis of randomized trials involving 11050 men. HIV medicine 2008; 9(6): 332-5.

50. Stringer EM, Sinkala M, Kumwenda R, et al. Personal risk perception, HIV knowledge and risk avoidance behavior, and their relationships to actual HIV serostatus in an urban African obstetric population. Journal of acquired immune deficiency syndromes (1999) 2004; 35(1): 60.

51. Econometric Software Inc. NLOGIT 5. 2012.

52. Volz E, Wejnert C, Deganii I, Heckathorn D. Respondent-Driven Sampling Analysis Tool (RDSAT) 6.0. New York: Cornell University; 2007.

53. Bech M, Gyrd-Hansen D. Effects coding in discrete choice experiments. Health economics 2005; 14(10): 1079-83.

54. Seutlwadi L, Peltzer K, Mchunu G, Tutshana BO. Contraceptive use and associated factors among South African youth (18-24 years): A population-based survey. South African Journal of Obstetrics and Gynaecology; 18(2).

55. Gomez GB, Borquez A, Case KK, Wheelock A, Vassall A, Hankins C. The Cost and Impact of Scaling Up Pre-exposure Prophylaxis for HIV Prevention: A Systematic Review of Cost-Effectiveness Modelling Studies. PLoS Med 2013; 10(3): e1001401.

56. Robinson J, Yeh E. Transactional Sex as a Response to Risk in Western Kenya. American Economic Journal-Applied Economics 2011; 3(1): 35-64.

57. Delany-Moretlwe S, Mullick S, Eakle R, Rees H. Planning for HIV preexposure prophylaxis introduction: lessons learned from contraception. Current opinion in HIV and AIDS 2016; 11(1): 87-93.

58. Quaife M, Terris-Prestholt F, Di Tanna GL, Vickerman P. PRM97 - Accounting for the Imperfect External Validity of Discrete Choice Experiments When Predicting Demand. Value in Health 2016; 19(7): A374.

59. Ford S, Stancil B, Markowitz M, et al. ECLAIR Study of Cabotegravir LA Injections: Characterization of Safety and PK During the" PK Tail" Phase. AIDS RESEARCH AND HUMAN RETROVIRUSES; 2016: MARY ANN LIEBERT, INC 140 HUGUENOT STREET, 3RD FL, NEW ROCHELLE, NY 10801 USA; 2016. p. 71-.

60. McCreesh N, Frost S, Seeley J, et al. Evaluation of Respondent-Driven Sampling. Epidemiology (Cambridge, Mass) 2012; 23(1): 138-47.

61. Department of Health South Africa, South African National AIDS Council. South African HIV and TB Investment Case - Summary Report Phase 1. Pretoria, 2016. 
